\title{
Statistical search for Be stars candidates in the Small Magellanic Cloud
}

\author{
Alejandro García-Varela ${ }^{1}$, Beatriz Sabogal ${ }^{1}$ and \\ Ronald E. Mennickent ${ }^{2}$ \\ ${ }^{1}$ Departamento de Física, Universidad de los Andes, Bogotá, Colombia \\ email: josegarc@uniandes.edu.co \\ ${ }^{2}$ Departamento de Astronomía, Facultad de Ciencias Físicas y Matemáticas, \\ Universidad de Concepción, Chile
}

\begin{abstract}
A report of a systematic search for Be star candidates in the Small Magellanic Cloud using statistical selection criteria is presented. The results are compared with those obtained with a standard photometric method to search for Be star candidates.
\end{abstract}

Keywords. methods: statistical, stars: variables: other, astronomical data bases: miscellaneous

\section{Introduction}

The standard method to search for variable stars inside photometric data bases like OGLE or MACHO implies that light curves are extracted and the mean magnitude and average standard deviation for each star are computed. Then, the stars are grouped and the average magnitude and average standard deviation are calculated per bin. The resulting pairs are plotted and fit with a polynomial, leading to a function $f$ representing the standard deviation as a function of mean magnitude. Stars with standard deviation values larger than a threshold value $(\alpha f)$ are picked out as variable star candidates. Visual inspection is needed to find the variable stars. The disadvantage of this method is that it consumes a lot of time inspecting light curves, while the discovery of new variable stars in a secure way is its main advantage.

\section{The statistical method}

During the OGLE II project (Udalski et al. 1997, Udalski et al. 2002), VI photometry maps of the Galactic Bulge (GB) and the Small Magellanic Cloud (SMC) were obtained. Within the OGLE II catalog of variable stars of the SMC we looked for stars with absolute $\mathrm{V}$ magnitudes in the typical range of the Galactic Be stars, i.e. $-6<M_{V}<0$ (Wegner 2000, Garmany \& Humphreys 1985), and with V-I colours in the range for classical Be stars. In order to search and find Be star candidates on the SMC, we established the following process:

1. To avoid noisy detections, we used a filter that rejected measurements larger than $2.5 \sigma$ times the mean I-band magnitude in all light curves.

2. We selected the stars with amplitudes $A_{I}$ between 0.05 mag (the limit of noise in OGLE II data) and 0.3 mag. Almost the $70 \%$ of the Be stars have amplitudes less than this upper limit (Sabogal et al. 2005).

3. We applied the statistical properties found for the GB Be stars to identify the Be star candidates in the SMC. The GB Be star candidates found by Sabogal et al. (2008) show a parabolic correlation between kurtosis excess $(K E)$ and skewness $(S)$ of the I magnitudes (Fig. 1). The skewness is a measure of the asymmetry of a distribution; for a 


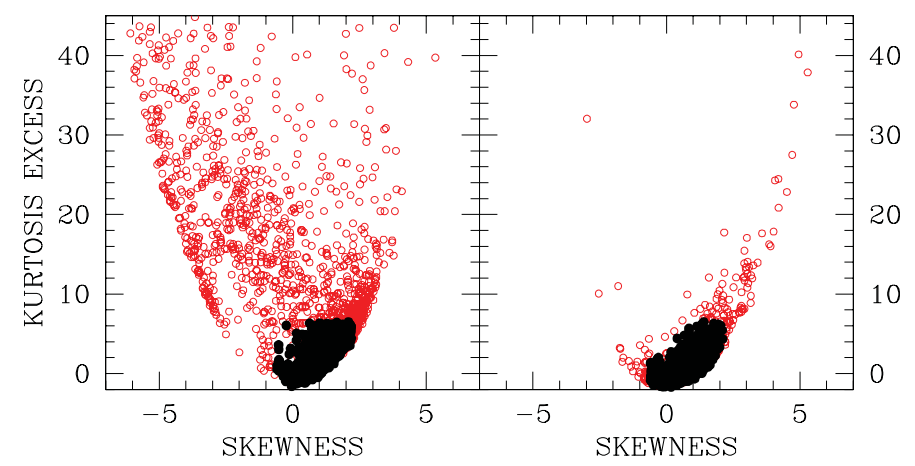

Figure 1. Statistical properties of GB (left) and SMC (right) stars. Open circles represent the variable stars candidates. Filled circles represent the Be star candidates.

Gaussian distribution $S$ is zero. Negative values of $S$ of a sample of data indicate that the left tail of the distribution is long relative to the right tail. Kurtosis $(K)$ is a measure of whether the distribution is sharp or flattened relative to a normal distribution. Datasets with high $K$ value tend to have a distinct peak relative to the mean and decline rather rapidly. Since $(K)$ of a normal distribution is 3 , we adopted $K E=K-3$ as the statistical variable to examine whether the distributions of I magnitudes are sharp.

Analyzing the parabolic behavior of the $K E$ vs $S$ diagram for GB variable star candidates, we found that the Be star candidates are located in the range $K E<6.5$ and $S$ between $[-0.6,2.2] . K E$ and $S$ of the I magnitudes of SMC variable stars also presented a parabolic behavior (Fig. 1), and the SMC stars falling in the range previously mentioned corresponded to the Be star candidates selected by standard photometric methods.

\section{Discussion and Conclusion}

5007 variable stars were selected of the OGLE II catalog of the SMC galaxy. Applying the statistical method described in previous section we found that the variable stars of the SMC show the same parabolic behavior in the $K E$ vs $S$ diagram than the variable stars of the GB. The locus of the GB Be stars in that correlation allowed us to find the Be stars of the SMC. This could imply that the range of $K E$ and $S$ found is valid for Be star candidates in different galaxies, and in that case this statistical method would be a useful method to search for Be stars candidates. We found 1623 candidates to Be stars in the SMC, most of them previously reported by Mennickent et al. (2002) in a search that used the traditional method. Those authors visually inspected 5007 light curves (LC) searching for Be stars, while, in our case, we only needed to inspect 1623 (only $32 \%$ of the LC inspected in the traditional method). We conclude that searching for Be star candidates is faster and more efficient using a statistical method than a traditional one.

\section{References}

Garmany, C. D. \& Humphreys, R. M. 1985, AJ, 90, 2009

Mennickent, R. E., Pietrzyński, G., Gieren, W., \& Szewczyk, O. 2002, A\&̛A, 393, 887

Sabogal, B. E., Mennickent, R. E., Pietrzyński, G. \& Gieren, W. 2005, MNRAS, 361, 1055

Sabogal, B. E., Mennickent, R. E., Pietrzyński, G., García, J. A. et al. 2008, A\& A, 478, 659

Udalski, A., Kubiak, M., \& Szymanski, M. 1997, AcA, 47, 319

Udalski, A., Szymanski, M., Kubiak, M., Pietrzynski, G. et al. 2002, AcA, 52, 217

Wegner, W. 2000, MNRAS, 319, 771 